\title{
Review Article \\ The Roles of Hedgehog Signaling in Upper Lip Formation
}

\author{
Hiroshi Kurosaka \\ Department of Orthodontics and Dentofacial Orthopedics, Graduate School of Dentistry, Osaka University, Osaka 565-0871, Japan
}

Correspondence should be addressed to Hiroshi Kurosaka; kurosaka@dent.osaka-u.ac.jp

Received 9 March 2015; Accepted 20 August 2015

Academic Editor: Sung-Hoon Kim

Copyright (c) 2015 Hiroshi Kurosaka. This is an open access article distributed under the Creative Commons Attribution License, which permits unrestricted use, distribution, and reproduction in any medium, provided the original work is properly cited.

\begin{abstract}
Craniofacial development consists of a highly complex sequence of the orchestrated growth and fusion of facial processes. It is also known that craniofacial abnormalities can be detected in $1 / 3$ of all patients with congenital diseases. Within the various craniofacial abnormalities, orofacial clefting is one of the most common phenotypic outcomes associated with retarded facial growth or fusion. Cleft lip is one of the representative and frequently encountered conditions in the spectrum of orofacial clefting. Despite various mechanisms or signaling pathways that have been proposed to be the cause of cleft lip, a detailed mechanism that bridges individual signaling pathways to the cleft lip is still elusive. Shh signaling is indispensable for normal embryonic development, and disruption can result in a wide spectrum of craniofacial disorders, including cleft lip. This review focuses on the current knowledge about the mechanisms of facial development and the etiology of cleft lip that are related to Shh signaling.
\end{abstract}

\section{Introduction}

The proper growth and fusion of embryonic facial processes are critical for craniofacial development, and failure of either step can lead to a wide variety of orofacial clefting. Cleft lip and/or palate $(\mathrm{CL} / \mathrm{P})$ is one of the most common orofacial clefts and is found in 1/700 living newborns [1]. CL/P has a lot of variation in terms of the degree of the cleft, such as cleft lip $(\mathrm{CL})$, cleft of the secondary palate (CP), and CL/P $[2,3]$. Most of the time, all of these cleft phenotypes are considered to be the same disorder with different severity, since the facial processes share a similar cellular context (mesenchymal cells surrounded by facial ectoderm). However, from the anatomical point of view, the lip and secondary palate have different origins, with the lip being formed by the fusion of medial and lateral nasal processes, while the secondary palate is a structure of fused palatal shelves that originate from maxillary processes. In addition, the timing of fusion is different between these two structures. For these reasons, it is worthwhile to consider the different cleft etiologies separately.

Many signaling pathways have been revealed to be associated with the etiology of CL/P [4]. Sonic hedgehog (Shh) signaling is one of the most important signaling pathways for the development of many organs, including craniofacial structures [5], and either a loss of function or gain of function of this signaling pathway can lead to craniofacial abnormalities, including CL/P [6, 7]. The mechanism of Shh signaling in secondary palate development has been well studied [8-11]; however, there have been a limited number of studies focused on lip development. In this review, we focus on and discuss the roles of Shh signaling during lip formation by summarizing the current knowledge based on many animal model studies including mice (Table 1).

\section{The Development of Facial Processes Required for Lip Formation}

In mice, the development of the medial nasal process (MNP) and lateral nasal process (LNP), which are the processes which eventually give rise to the nose and part of the lip, are not visibly evident on embryonic day (E)10.0, and the frontonasal process (FNP) still has a relatively flat structure (Figures 1(a) and 1(f)). After around E10.5, the MNP and LNP start to grow out from the FNP (Figures $1(\mathrm{~b})$ and $1(\mathrm{~g})$ ). These processes continue to grow and begin to fuse at the position called the lambdoidal region (Figures $1(\mathrm{c})-1(\mathrm{e})$ and $1(\mathrm{~h})-1(\mathrm{j})$, red arrowhead) where MNP, LNP, and maxillary process (MXP) integrate together (Figures $1(\mathrm{c})-1(\mathrm{e})$ and $1(\mathrm{~h})-$ $1(j))$. The growth and fusion of these developing processes 

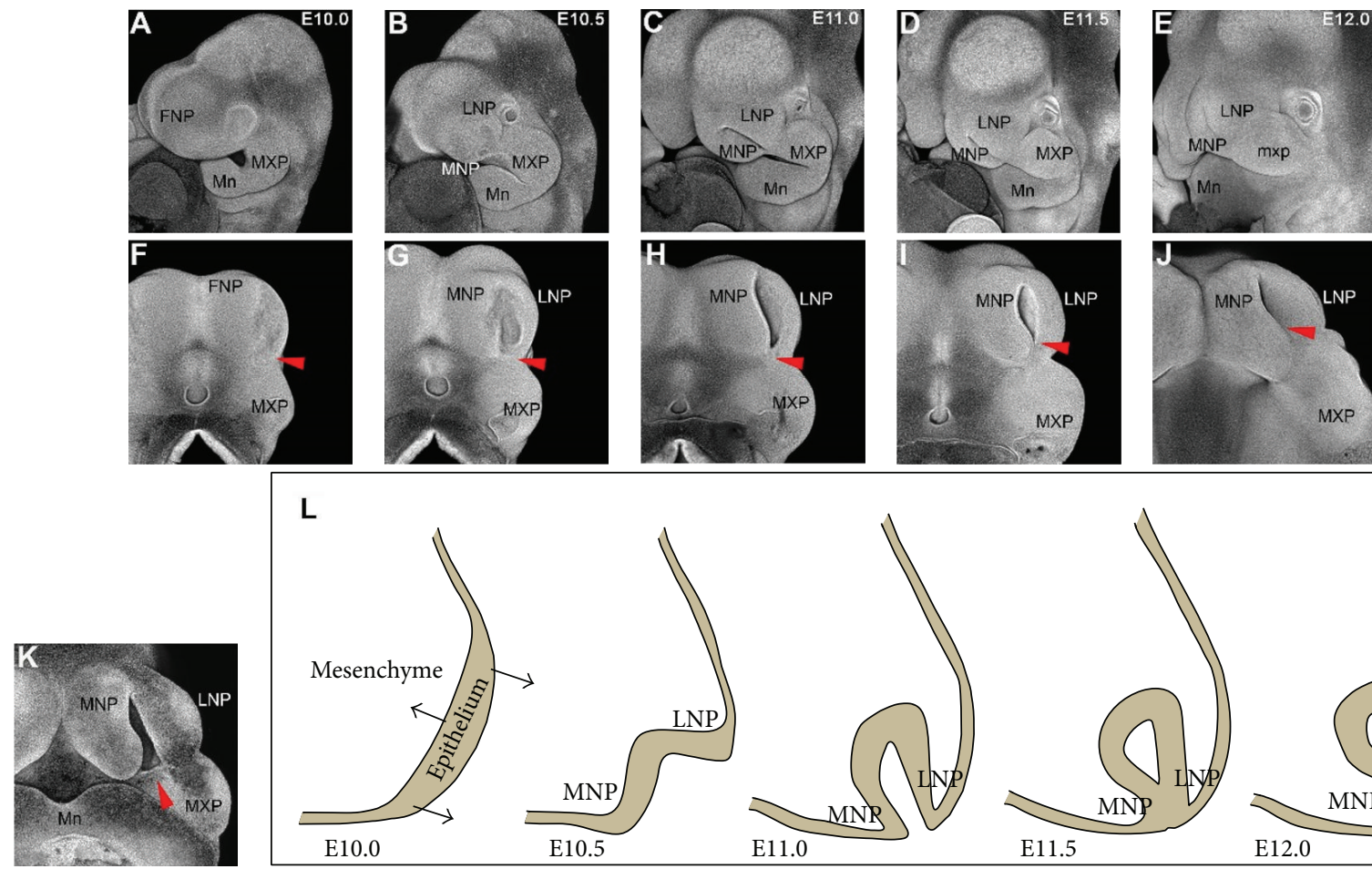

$\mathbf{L}$
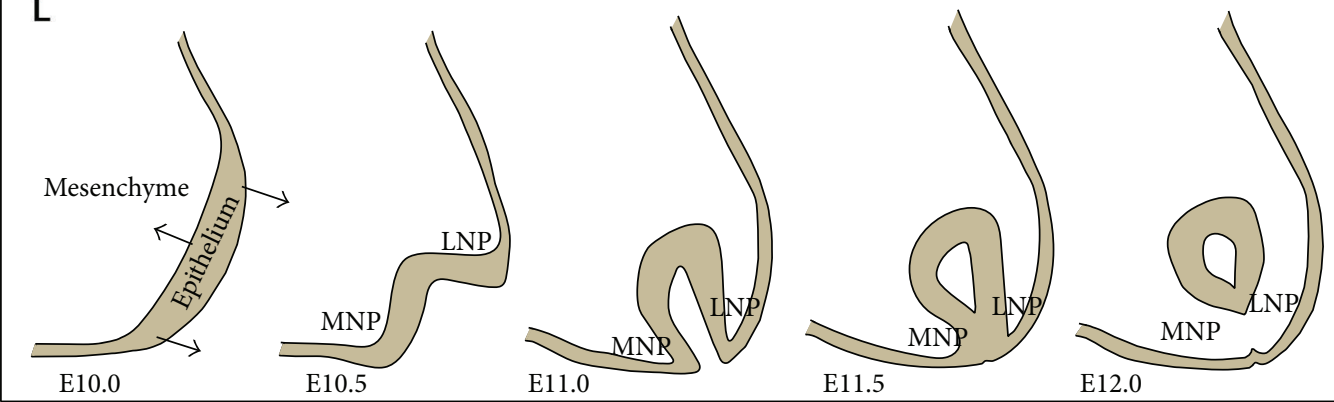

Figure 1: The growth of facial processes in mouse embryos shown by whole mount nuclear fluorescent imaging. ((A)-(E)) Oblique views of the facial development of mouse embryos from E10 to E12.0. ((F)-(J)) The same heads were captured from the ventral side of the head with the mandible removed. (K) The representative phenotype of cleft lip, which was induced by whole embryo culture. The red arrowheads show the position where the facial processes fuse. (L) Cartoon sequence of growing nasal processes at each stage. Black arrow shows the direction of processes growth and folding. MNP: medial nasal process, LNP: lateral nasal process, MXP: maxillary process, and Mn; mandible.

TABLE 1: Mouse mutants that were used for investigating Shh signaling and lip development.

\begin{tabular}{|c|c|c|c|}
\hline Mutated gene & Type of mutation & Phenotype & Reference \\
\hline Patched1 & ENU & $\mathrm{CL}$ & [7] \\
\hline Patched1 & $\begin{array}{l}\text { CKO (neural } \\
\text { crest) }\end{array}$ & $\mathrm{CL}$ & {$[33]$} \\
\hline Smo & $\begin{array}{l}\text { CKO (neural } \\
\text { crest) }\end{array}$ & Truncated face & {$[35]$} \\
\hline Shh & $\begin{array}{c}\text { Ectoderm } \\
\text { overexpression }\end{array}$ & Malformed lip & {$[36]$} \\
\hline Boc, Gas1 & Double KO & $\mathrm{CL} / \mathrm{P}$ & {$[31]$} \\
\hline$K i f 3 a$ & $\begin{array}{l}\text { CKO (neural } \\
\text { crest) }\end{array}$ & Hypertelorism, CP & {$[41]$} \\
\hline Ift172 & ENU & $\mathrm{CP}$ & {$[42]$} \\
\hline Ift144 & ENU & $\mathrm{CL} / \mathrm{P}$ & {$[44]$} \\
\hline
\end{tabular}

ENU: $N$-ethyl- $N$-nitrosourea. CKO: conditional knockout. KO: knockout. CL: cleft lip. CP: cleft palate. CL/P: cleft lip and palate.

are crucial for normal midface development, including lip formation. If these processes fail to fuse, it can lead to CL (Figure 1(k)). Several studies have shown that the surgical removal or mechanical inhibition of facial processes results in cleft lip in rat embryos $[12,13]$. Interestingly, the growth and fusion of the facial processes at these stages seem to be largely evolutionally conserved among many species, which makes animal models useful for investigating the mechanisms underlying human cleft lip [14-16].

\section{The Role of Shh Signaling in Removing Epithelial Seam Cells}

At the lambdoidal region, the epithelial seam cells between fusing processes have to break down in order to form a mesenchymal bridge (Figure 1(l)). A few mechanisms have been proposed to explain this breakdown of the epithelial seam, such as apoptosis or the epithelial mesenchymal transition (EMT). Similar to fusion of the secondary palate, active cell death can be observed in the epithelial seam between the fusing MNP and LNP in mice [7, 14]. However, mice that lacked this apoptosis due to inhibition of the caspase signaling pathway did not show CL but did have secondary palate malformation and exencephaly $[17,18]$. These results suggested that there are other mechanisms leading to the removal of the epithelial seam cells. Some studies have shown that the EMT occurs in epithelial seam cells in chick fusing facial processes [19]. However, there have been no experiments that have inhibited the EMT completely from these fusing processes, so the mechanism by which the epithelial seam cells are removed is not fully understood.

Another epithelial cell that plays a critical role for fusing facial processes is the periderm cells, which slough off from the growing processes to allow for proper fusion [20]. 


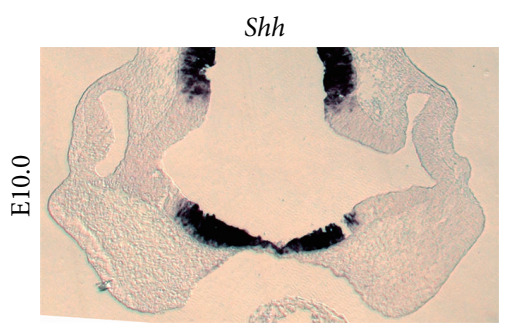

(a)

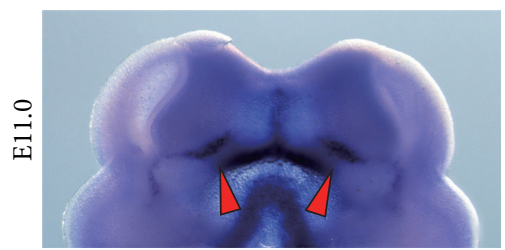

(c)

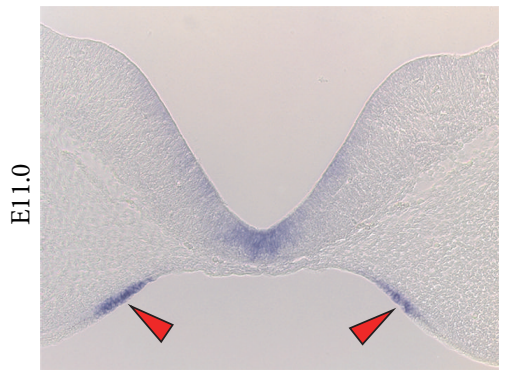

(e)

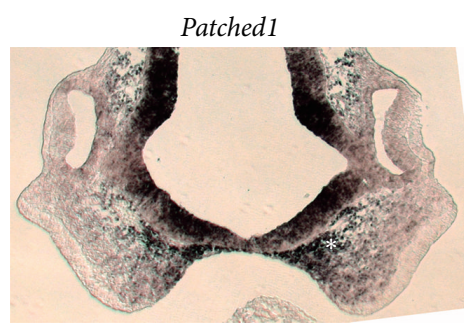

(b)

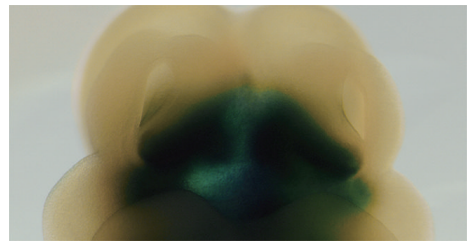

(d)

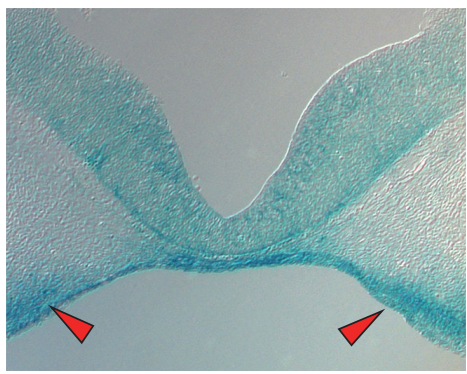

(f)

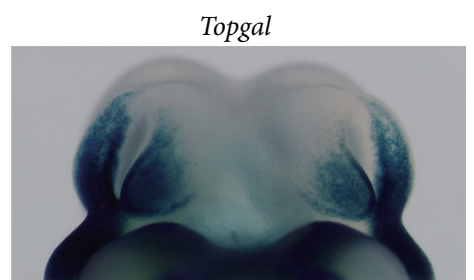

(g)

FIgURE 2: The expression of Shh and Patchedl during the growth of facial processes in mouse embryos. ((a), (c), and (e)) In situ hybridization of Shh on E10 and E11.0. (b) In situ hybridization of Patched1 on E10. ((d) and (f)) LacZ staining of E11.0 Patched1-LacZ mice. (g) LacZ staining of a Topgal mouse embryo on E11.0. The red arrowheads indicate the position of the frontonasal ectodermal zone (FEZ).

In the mouse secondary palate, synergistic mutations of $P 63$ and Irf6 lead to excessive layers of periderm cells, which results in cleft palate $[21,22]$. On the other hand, Irf6 null mice showed a lack of periderm cells, which was shown to be associated with ectopic fusion of the secondary palatal shelf to the tongue [23]. Additionally, Irf6 was shown to allow the epithelial cells to exit the cell cycle in order to remove the epithelium [21]. In our previous study, we discovered that a disturbed $S h h$ signaling gradient resulted in perturbed P63 and Irf6 expression, which possibly led to persistent SSEA1-positive periderm cells on the MNP, resulting in the $\mathrm{CL}$ phenotype, together with alterations in the balance of cell proliferation and apoptosis at the epithelial seam, which caused a failure in the removal of the epithelial seam [7]. However, the relationship between Shh signaling and periderm cell development and the relationship between periderm cell defects and the etiology of CL are still elusive and require further studies.

\section{The Expression and Role of Shh Signaling during the Growth and Fusion of Facial Processes}

Shh signaling plays various important roles in craniofacial development. In addition, the expression of Shh shows a unique pattern during the outgrowth and fusion of nasal processes. Before evident outgrowth of the MNP and LNP, Shh is expressed at the ventral neural tube, but not at the nasal process (Figure 2(a)). At the same stage, mesenchymal cells in the frontonasal processes are already receiving the $\mathrm{SHH}$ ligand to activate the Hedgehog ( $\mathrm{Hh}$ ) signaling pathway, as shown by Patched 1 expression, which is one of the receptors and markers of $H h$ signaling (Figure 2(b), asterisk). Patched1 expression becomes stronger at the center of the face, which may be caused by the high amount of SHH ligand secreted from the ventral neural tube. Around E11.0 in mice, Shh expression started to be observed at specific regions of the oral ectoderm, followed by expanded Patched 1 expression, mainly in the MNP (Figures 2(c)-2(f)). It is well known that this intraoral Shh-expressing region, together with the Fgf8 expressing domain, plays a critical role in the growth and patterning of the FNP, which is called the frontonasal ectodermal zone (FEZ) [24, 25]. The Shh expression at the FEZ region is known to require Shh signaling from the ventral neural tube and Bmp signaling from the FNP [26,27]. Genetic ablation of Shh during mouse development tended to lead to a smaller MNP or diminished MNP development, which was associated with severe cell death and holoprosencephaly, while the LNP developed relatively normally, as shown by analyzing molecular markers such as $\operatorname{Pax} 9$ or $\operatorname{Pax} 7$ [28-30]. 
Synergistic mutations in Boc and Gasl, which are receptors of $\mathrm{SHH}$ ligand, led to CL with holoprosencephaly [31]. In addition, the prenatal administration of a Shh signaling inhibitor could cause a similar phenotype, with wide variation $[6,32]$.

On the other hand, enhanced Shh signaling due to mutated Patched 1 during head development could also result in CL with severe craniofacial abnormalities, including hypertelorism [7]. These results, in conjunction with the induction of CL by surgical removal of facial prominences in rat embryos [12], indicate that adequate MNP development, governed by proper Shh signaling from the ventral neural tube and consequently at the FEZ region, is essential for the fusion of facial processes and lip formation. It is also important to understand the tissue-specific roles of Shh signaling during lip fusion. Mutant mice that had Patched1 conditionally knocked out from cranial neural crest cells also showed a CL phenotype [33].

Smoothened (Smo) is another critical mediator of Shh signaling [34]. A previous study showed that inhibiting the activity of Smo in cranial neural crest cells was associated with craniofacial abnormalities, such as a dramatically truncated face, which included a severely deformed lip, together with hypoplastic cranial bones [35]. Conversely, the overexpression of Shh in the facial ectoderm was also associated with lip abnormalities with CP [36]. These results indicate that adequate Shh signaling in both the developing facial ectoderm and neural crest cells is essential for proper lip formation. Subsequently, Shh also starts to be expressed at the ventral nasal pit epithelium while the MNP and LNP are fusing $[35,37,38]$. The role of this Shh signaling in lip formation is still unclear and requires further investigation by removing or enhancing Shh signaling in a tissue- and stagespecific manner.

\section{Cilia-Associated Shh Signaling and Midfacial Development}

Primary cilia are thin cellular processes that extend from the surface of various types of cells. It is well known that cilia have important roles in a variety of signaling pathways which are indispensable for proper development or metabolism [39]. Primary cilia are known to work as mediators of Shh signaling by analyzing the localization of PATCHED1 and SMOOTHENED, which are the important downstream factors required for activating Shh signaling in response to the ligand [34]. In humans, there is a disease spectrum of ciliopathy caused by mutations of the genes important for ciliogenesis, such as intraflagellar transport proteins (IFTs). These exhibit a wide spectrum of phenotypes, including craniofacial defects $[39,40]$. Previous reports showed multiple mice mutants that had disrupted ciliary proteins that were associated with craniofacial abnormalities.

Interestingly, many of these mutants had phenotypes that could have resulted from disrupting Shh signaling, such as polydactyly or anencephaly. Neural crest-specific elimination of Kif $3 a$, one of the intraflagellar transport proteins in mice, caused excessive Shh signaling with hypertelorism and a midfacial cleft [41]. Mutation of Ift172 in mice leads to CP and recapitulates the phenotype of human VACTERL $[42,43]$. Disrupting Ift144 led to CL with anencephaly and polydactyly, which are representative phenotypes of disrupted Shh signaling [44]. Interestingly, many phenotypes of this mutant resembled those of a compound mutant that we reported to have a disrupted gradient of Shh signaling [7]. These results suggest that cilia play important roles to produce a specific gradient of Shh signaling.

In avian species, there are several well-studied naturally occurring mutants named talpids (talpid, talpid ${ }^{2}$, and talpid ${ }^{3}$ ), which exhibit various developmental defects, including craniofacial abnormalities $[45,46]$. In particular, talpid ${ }^{2}$ shows bilateral clefting between the frontonasal process and LNP, and a causative mutation has been identified in the C2CD3 gene, which is important for ciliogenesis [46]. These results indicate that Shh signaling, which is mediated by cilia, is essential for lip formation. However, it is currently unknown how different cilial proteins can affect craniofacial development. In addition, there are different signaling pathways which are known to be regulated by primary cilia [47], and further comprehensive analyses are necessary to link the cilial defects to craniofacial disorders like CL.

\section{Critical Interaction between Shh and Wnt Signaling During Lip Fusion}

Canonical Wnt signaling is indispensable for normal facial development, including lip fusion. In both humans and mice, disruption of Wnt signaling has been shown to cause CL/P [48-50]. Additionally, the P63-Irf6 signaling pathway has been identified to be activated by canonical Wnt signaling, which was associated with the growth and fusion of facial processes growth and fusion [51]. In our recent study, we discovered that Shh signaling (Ptch1-LacZ) and canonical Wnt signaling (Topgal) showed a complementary expression pattern during craniofacial development (Figures 2(c), 2(d), and 2(g)). Furthermore, we proved that enhanced Shh signaling could result in CL by inhibiting canonical Wnt signaling [7]. Previous research showed that genetic elimination of Kif $3 a$ from cranial neural crest cells in mice led to enhanced Shh signaling, together with disturbed canonical Wnt signaling in a tissue- and time-dependent manner [41]. These results strongly suggest that there is a critical interaction between Shh and canonical Wht signaling during craniofacial development, and this is indispensable for proper lip formation.

\section{Ethanol Exposure and Shh Signaling Affect Lip Formation}

The etiology of CL is known to include both genetic and environmental factors [52]. One of the most well-studied maternal environmental factors that can lead to craniofacial abnormalities is ethanol exposure. Ethanol exposure of the developing embryo is known to cause craniofacial and brain deformities which resemble the phenotype of disrupted Shh signaling [53]. There are reports showing that embryonic ethanol exposure disrupts Shh signaling and enhances cell 
death in the prechordal mesendoderm and cranial neural crest cells $[28,54]$. Recent studies showed the interaction between Shh signaling and ethanol exposure by demonstrating a significantly enhanced phenotype in Shh or Gli2 heterozygous knockout mice that were treated with ethanol [55]. There was another report that showed that disruption of Cdon, one of the receptors for Shh signaling, together with ethanol exposure, would result into holoprosencephaly, while Cdon knockout mice did not exhibit this phenotype without the environmental insult [56]. This synergistic effect with Cdon could be restored by removing one copy of Patched 1 [57]. These results clearly demonstrate that there is a molecular and environmental interaction between Shh signaling and ethanol exposure during craniofacial development.

\section{Conflict of Interests}

The author declares that there are no conflict of interests regarding the publication of this paper.

\section{Acknowledgments}

The author sincerely appreciates Dr. Paul A. Trainor (Stowers Institute for Medical Research) for all of the discussions and the help with writing this review. This work was partially supported by Grant-in-Aid for Young Scientists (A) (to Hiroshi Kurosaka).

\section{References}

[1] M. J. Dixon, M. L. Marazita, T. H. Beaty, and J. C. Murray, "Cleft lip and palate: understanding genetic and environmental influences," Nature Reviews Genetics, vol. 12, no. 3, pp. 167-178, 2011.

[2] Y. Nagase, N. Natsume, T. Kato, and T. Hayakawa, "Epidemiological analysis of cleft lip and/or palate by cleft pattern," Journal of Maxillofacial and Oral Surgery, vol. 9, no. 4, pp. 389-395, 2010.

[3] K. H. Wang, C. L. Heike, M. D. Clarkson et al., "Evaluation and integration of disparate classification systems for clefts of the lip," Frontiers in Physiology, vol. 5, article 163, 2014.

[4] E. J. Leslie and M. L. Marazita, "Genetics of cleft lip and cleft palate," American Journal of Medical Genetics C: Seminars in Medical Genetics, vol. 163, no. 4, pp. 246-258, 2013.

[5] M. Varjosalo and J. Taipale, "Hedgehog: functions and mechanisms," Genes \& Development, vol. 22, no. 18, pp. 2454-2472, 2008.

[6] R. J. Lipinski, C. Song, K. K. Sulik et al., "Cleft lip and palate results from Hedgehog signaling antagonism in the mouse: phenotypic characterization and clinical implications," Birth Defects Research Part A: Clinical and Molecular Teratology, vol. 88, no. 4, pp. 232-240, 2010.

[7] H. Kurosaka, A. Iulianella, T. Williams, and P. A. Trainor, "Disrupting hedgehog and WNT signaling interactions promotes cleft lip pathogenesis," The Journal of Clinical Investigation, vol. 124, no. 4, pp. 1660-1671, 2014.

[8] R. Rice, B. Spencer-Dene, E. C. Connor et al., "Disruption of Fgf10/Fgfr2b-coordinated epithelial-mesenchymal interactions causes cleft palate," The Journal of Clinical Investigation, vol. 113, no. 12, pp. 1692-1700, 2004.
[9] Y. Lan and R. Jiang, "Sonic hedgehog signaling regulates reciprocal epithelial-mesenchymal interactions controlling palatal outgrowth," Development, vol. 136, no. 8, pp. 1387-1396, 2009.

[10] M. T. Cobourne and J. B. Green, "Hedgehog signalling in development of the secondary palate," Frontiers of Oral Biology, vol. 16, pp. 52-59, 2012.

[11] M. Seppala, M. J. Depew, D. C. Martinelli, C. Fan, P. T. Sharpe, and M. T. Cobourne, "Gas1 is a modifier for holoprosencephaly and genetically interacts with sonic hedgehog," The Journal of Clinical Investigation, vol. 117, no. 6, pp. 1575-1584, 2007.

[12] N. Ohbayashi and K. Eto, "Relative contributions of the facial processes to facial development: a microsurgical assay," Journal of Craniofacial Genetics and Developmental Biology, vol. 6, no. 2, pp. 41-44, 1986.

[13] K. Eto, A. Figueroa, G. Tamura, and R. M. Pratt, "Induction of cleft lip in cultured rat embryos by localized administration of tunicamycin," Journal of Embryology and Experimental Morphology, vol. 64, pp. 1-9, 1981.

[14] R. Jiang, J. O. Bush, and A. C. Lidral, "Development of the upper lip: morphogenetic and molecular mechanisms," Developmental Dynamics, vol. 235, no. 5, pp. 1152-1166, 2006.

[15] T. C. Cox, "Taking it to the max: the genetic and developmental mechanisms coordinating midfacial morphogenesis and dysmorphology," Clinical Genetics, vol. 65, no. 3, pp. 163-176, 2004.

[16] N. M. Young, D. Hu, A. J. Lainoff et al., "Embryonic bauplans and the developmental origins of facial diversity and constraint," Development, vol. 141, no. 5, pp. 1059-1063, 2014.

[17] N. Honarpour, C. Du, J. A. Richardson, R. E. Hammer, X. Wang, and J. Herz, "Adult Apaf-1-deficient mice exhibit male infertility," Developmental Biology, vol. 218, no. 2, pp. 248-258, 2000.

[18] J.-Z. Jin and J. Ding, "Analysis of cell migration, transdifferentiation and apoptosis during mouse secondary palate fusion," Development, vol. 133, no. 17, pp. 3341-3347, 2006.

[19] D. Sun, S. Baur, and E. D. Hay, "Epithelial-mesenchymal transformation is the mechanism for fusion of the craniofacial primordia involved in morphogenesis of the chicken lip," Developmental Biology, vol. 228, no. 2, pp. 337-349, 2000.

[20] R. J. Richardson, J. Dixon, R. Jiang, and M. J. Dixon, "Integration of IRF6 and Jagged 2 signalling is essential for controlling palatal adhesion and fusion competence," Human Molecular Genetics, vol. 18, no. 14, pp. 2632-2642, 2009.

[21] F. Moretti, B. Marinari, N. Lo Iacono et al., "A regulatory feedback loop involving p63 and IRF6 links the pathogenesis of 2 genetically different human ectodermal dysplasias," The Journal of Clinical Investigation, vol. 120, no. 5, pp. 1570-1577, 2010.

[22] H. A. Thomason, H. Zhou, E. N. Kouwenhoven et al., "Cooperation between the transcription factors p63 and IRF6 is essential to prevent cleft palate in mice," The Journal of Clinical Investigation, vol. 120, no. 5, pp. 1561-1569, 2010.

[23] R. J. Richardson, N. L. Hammond, P. A. Coulombe et al., "Periderm prevents pathological epithelial adhesions during embryogenesis," Journal of Clinical Investigation, vol. 124, no. 9, pp. 3891-3900, 2014.

[24] D. Hu, R. S. Marcucio, and J. A. Helms, "A zone of frontonasal ectoderm regulates patterning and growth in the face," Development, vol. 130, no. 9, pp. 1749-1758, 2003.

[25] D. Hu and R. S. Marcucio, "Unique organization of the frontonasal ectodermal zone in birds and mammals," Developmental Biology, vol. 325, no. 1, pp. 200-210, 2009. 
[26] D. Hu and R. S. Marcucio, "A SHH-responsive signaling center in the forebrain regulates craniofacial morphogenesis via the facial ectoderm," Development, vol. 136, no. 1, pp. 107-116, 2008.

[27] S. Foppiano, D. Hu, and R. S. Marcucio, "Signaling by bone morphogenetic proteins directs formation of an ectodermal signaling center that regulates craniofacial development," Developmental Biology, vol. 312, no. 1, pp. 103-114, 2007.

[28] K. Aoto, Y. Shikata, H. Imai et al., "Mouse Shh is required for prechordal plate maintenance during brain and craniofacial morphogenesis," Developmental Biology, vol. 327, no. 1, pp. 106120, 2009.

[29] J. F. Dennis, H. Kurosaka, A. Iulianella et al., "Mutations in Hedgehog acyltransferase (Hhat) perturb Hedgehog signaling, resulting in severe acrania-holoprosencephaly-agnathia craniofacial defects," PLoS Genetics, vol. 8, no. 10, Article ID e1002927, 2012.

[30] K. Aoto and P. A. Trainor, "Co-ordinated brain and craniofacial development depend upon Patched1/XIAP regulation of cell survival," Human Molecular Genetics, vol. 24, no. 3, pp. 698-713, 2015.

[31] M. Seppala, G. M. Xavier, C. Fan, and M. T. Cobourne, "Boc modifies the spectrum of holoprosencephaly in the absence of Gasl function," Biology Open, vol. 3, no. 8, pp. 728-740, 2014.

[32] R. J. Lipinski, P. R. Hutson, P. W. Hannam et al., "Dose- and route-dependent teratogenicity, toxicity, and pharmacokinetic profiles of the hedgehog signaling antagonist cyclopamine in the mouse," Toxicological Sciences, vol. 104, no. 1, pp. 189-197, 2008.

[33] V. Metzis, A. D. Courtney, M. C. Kerr et al., "Patched1 is required in neural crest cells for the prevention of orofacial clefts," Human Molecular Genetics, vol. 22, no. 24, pp. 50265035, 2013.

[34] R. Rohatgi, L. Milenkovic, and M. P. Scott, "Patched1 regulates hedgehog signaling at the primary cilium," Science, vol. 317, no. 5836, pp. 372-376, 2007.

[35] J. Jeong, J. Mao, T. Tenzen, A. H. Kottmann, and A. P. McMahon, "Hedgehog signaling in the neural crest cells regulates the patterning and growth of facial primordia," Genes \& Development, vol. 18, no. 8, pp. 937-951, 2004.

[36] M. T. Cobourne, G. M. Xavier, M. Depew et al., "Sonic hedgehog signalling inhibits palatogenesis and arrests tooth development in a mouse model of the nevoid basal cell carcinoma syndrome," Developmental Biology, vol. 331, no. 1, pp. 38-49, 2009.

[37] D. Hu, N. M. Young, X. Li, Y. Xu, B. Hallgrımsson, and R. S. Marcucio, "A dynamic shh expression pattern, regulated by shh and bmp signaling, coordinates fusion of primordia in the amniote face," Development, vol. 142, no. 3, pp. 567-574, 2015.

[38] G. M. Xavier, L. Panousopoulos, and M. T. Cobourne, "Scube3 is expressed in multiple tissues during development but is dispensable for embryonic survival in the mouse," PLOS ONE, vol. 8, no. 1, Article ID e55274, 2013.

[39] S. C. Goetz and K. V. Anderson, "The primary cilium: a signalling centre during vertebrate development," Nature Reviews Genetics, vol. 11, no. 5, pp. 331-344, 2010.

[40] P. J. Ocbina, J. T. Eggenschwiler, I. Moskowitz, and K. V. Anderson, "Complex interactions between genes controlling trafficking in primary cilia," Nature Genetics, vol. 43, no. 6, pp. 547-553, 2011.

[41] S. A. Brugmann, N. C. Allen, A. W. James, Z. Mekonnen, E. Madan, and J. A. Helms, "A primary cilia-dependent etiology for midline facial disorders," Human Molecular Genetics, vol. 19, no. 8, pp. 1577-1592, 2010.
[42] J. M. Friedland-Little, A. D. Hoffmann, P. J. Ocbina et al., "A novel murine allele of intraflagellar transport protein 172 causes a syndrome including VACTERL-like features with hydrocephalus," Human Molecular Genetics, vol. 20, no. 19, pp. 3725-3737, 2011.

[43] S. A. Temtamy and J. D. Miller, "Extending the scope of the VATER association: definition of the VATER syndrome," The Journal of Pediatrics, vol. 85, no. 3, pp. 345-349, 1974.

[44] A. Ashe, N. C. Butterfield, L. Town et al., "Mutations in mouse Ift144 model the craniofacial, limb and rib defects in skeletal ciliopathies," Human Molecular Genetics, vol. 21, no. 8, pp. 18081823, 2012.

[45] P. Buxton, M. G. Davey, I. R. Paton et al., "Craniofacial development in the talpid 3 chicken mutant," Differentiation, vol. 72, no. 7, pp. 348-362, 2004.

[46] C. Chang, E. N. Schock, E. A. O’Hare et al., “The cellular and molecular etiology of the craniofacial defects in the avian ciliopathic mutant talpid2," Development, vol. 141, no. 15, pp. 3003-3012, 2014.

[47] J. E. Lee and J. G. Gleeson, "Cilia in the nervous system: linking cilia function and neurodevelopmental disorders," Current Opinion in Neurology, vol. 24, no. 2, pp. 98-105, 2011.

[48] B. T. Chiquet, S. H. Blanton, A. Burt et al., "Variation in WNT genes is associated with non-syndromic cleft lip with or without cleft palate," Human Molecular Genetics, vol. 17, no. 14, pp. 22122218, 2008.

[49] L. Song, Y. Li, K. Wang et al., "Lrp6-mediated canonical Wnt signaling is required for lip formation and fusion," Development, vol. 136, no. 18, pp. 3161-3171, 2009.

[50] Y.-R. Jin, X. H. Han, M. M. Taketo, and J. K. Yoon, "Wnt9bdependent FGF signaling is crucial for outgrowth of the nasal and maxillary processes during upper jaw and lip development," Development, vol. 139, no. 10, pp. 1821-1830, 2012.

[51] E. Ferretti, B. Li, R. Zewdu et al., "A conserved Pbx-Wntp63-Irf6 regulatory module controls face morphogenesis by promoting epithelial apoptosis," Developmental Cell, vol. 21, no. 4, pp. 627-641, 2011.

[52] A. R. Vieira, "Genetic and environmental factors in human cleft lip and palate," Frontiers of Oral Biology, vol. 16, pp. 19-31, 2012.

[53] R. J. Lipinski, P. Hammond, S. K. O'Leary-Moore et al., "Ethanol-induced face-brain dysmorphology patterns are correlative and exposure-stage dependent," PLoS ONE, vol. 7, no. 8, Article ID e43067, 2012.

[54] S. C. Ahlgren, V. Thakur, and M. Bronner-Fraser, "Sonic hedgehog rescues cranial neural crest from cell death induced by ethanol exposure," Proceedings of the National Academy of Sciences, vol. 99, no. 16, pp. 10476-10481, 2002.

[55] H. W. Kietzman, J. L. Everson, K. K. Sulik, and R. J. Lipinski, "The teratogenic effects of prenatal ethanol exposure are exacerbated by sonic Hedgehog or Gli2 haploinsufficiency in the mouse," PLoS ONE, vol. 9, no. 2, Article ID e89448, 2014.

[56] M. Hong and R. S. Krauss, "Cdon mutation and fetal ethanol exposure synergize to produce midline signaling defects and holoprosencephaly spectrum disorders in mice," PLoS Genetics, vol. 8, no. 10, Article ID e1002999, 2012.

[57] M. Hong and R. S. Krauss, "Rescue of holoprosencephaly in fetal alcohol-exposed Cdon mutant mice by reduced gene dosage of Ptchl," PLoS ONE, vol. 8, no. 11, Article ID e79269, 2013. 

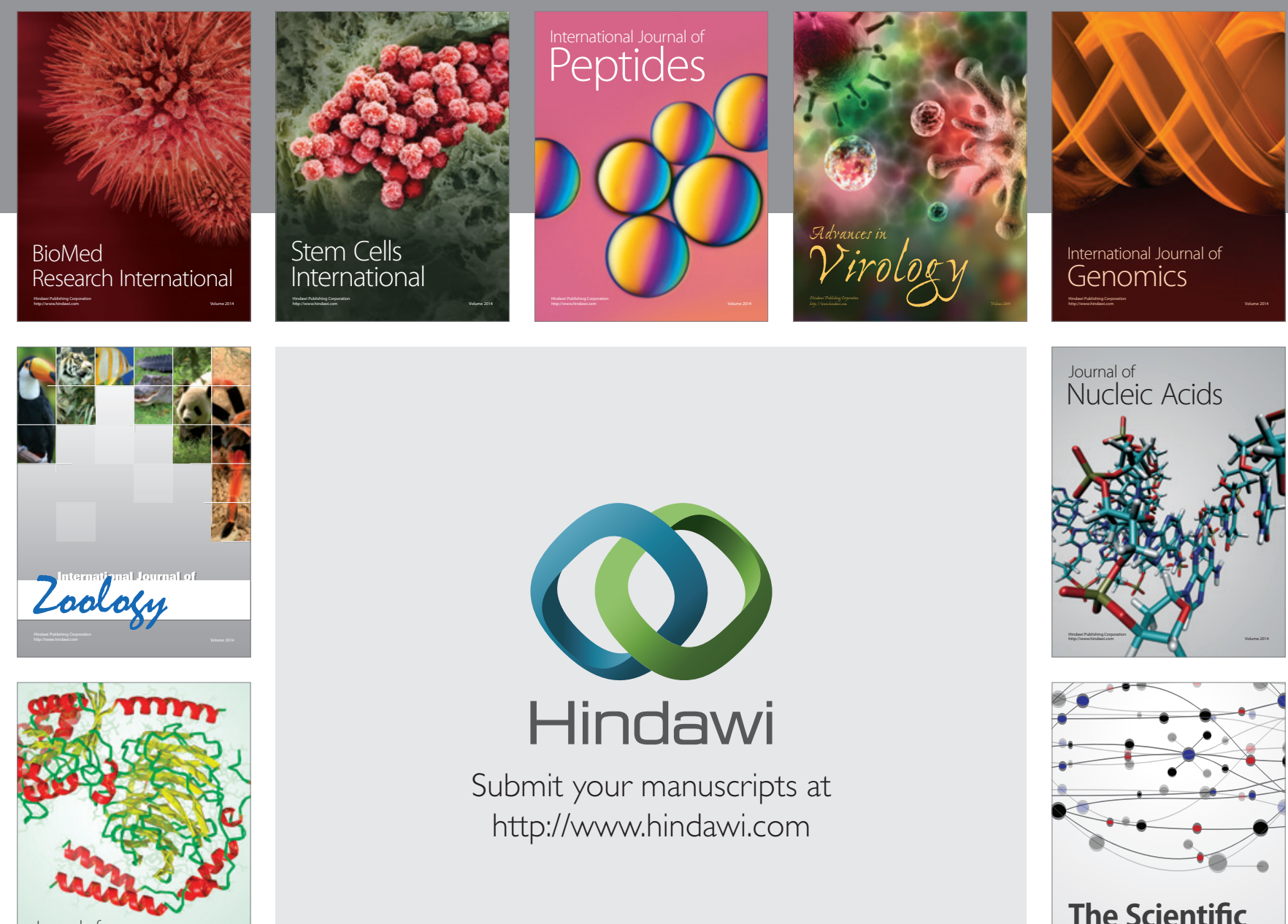

Submit your manuscripts at

http://www.hindawi.com

Journal of
Signal Transduction
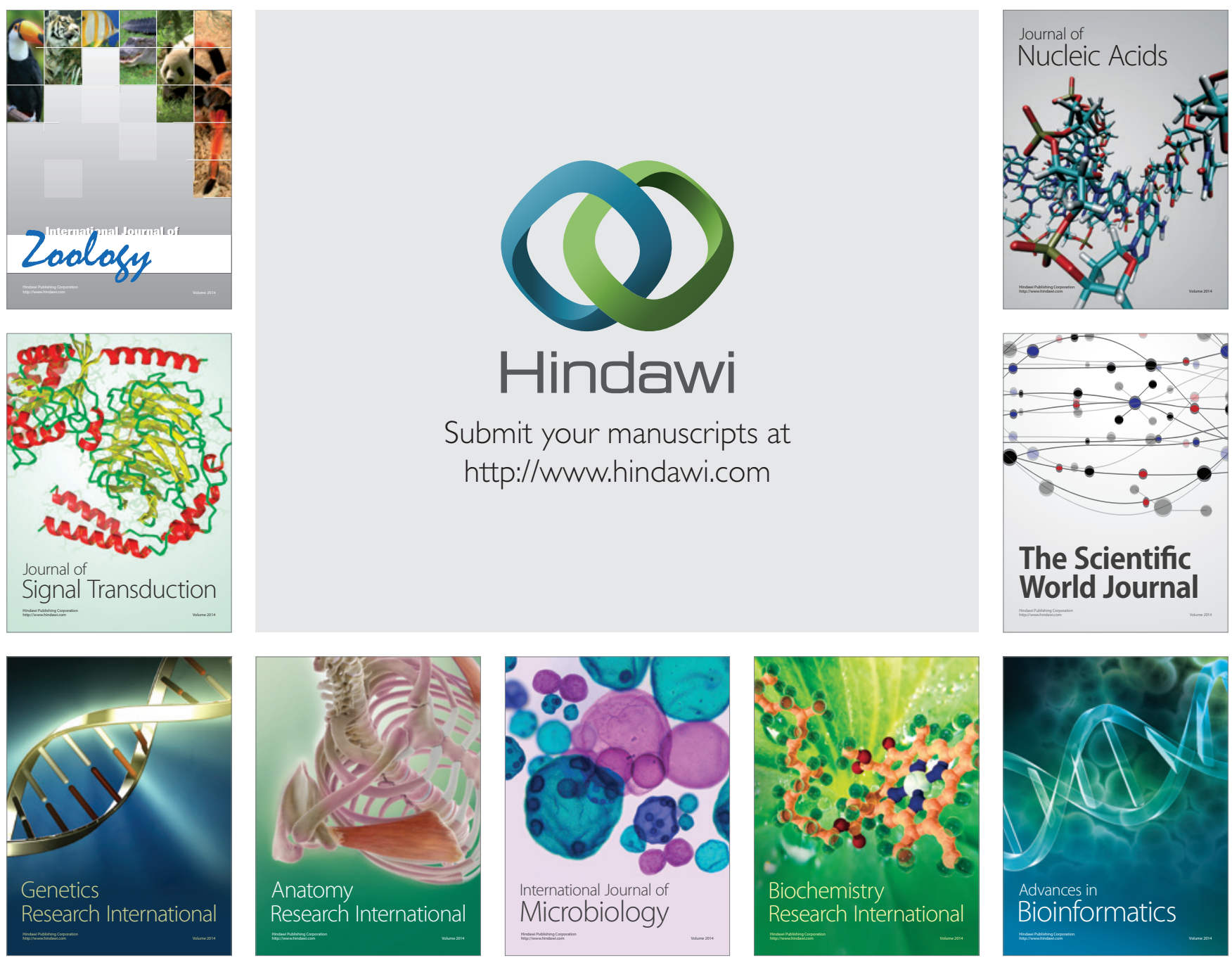

The Scientific World Journal
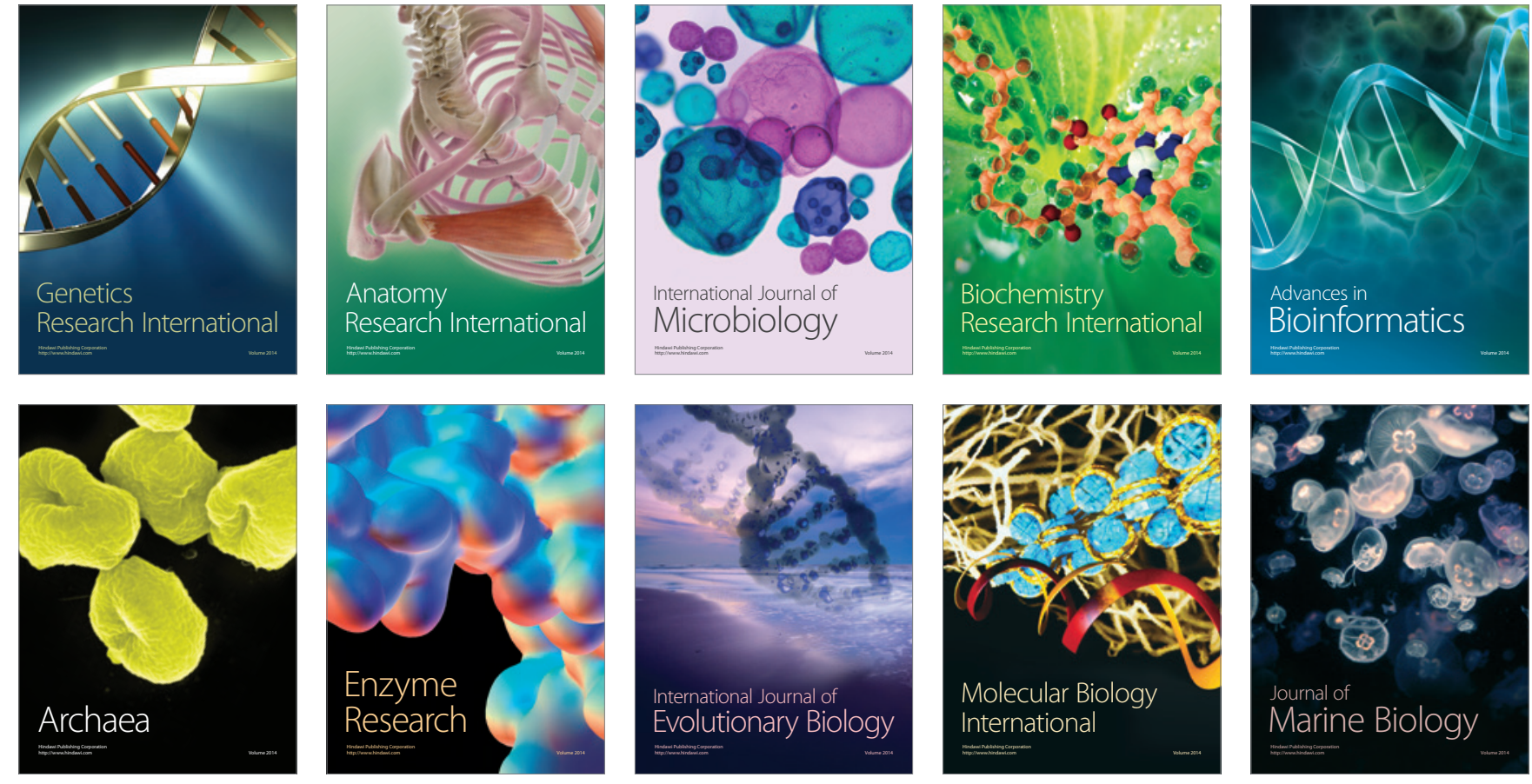